\title{
Large and symmetric deflection of Acryl plate-CFRP plate laminate actuator
}

\author{
Wenyi Lin, Minoru Sasaki* and Hirohisa Tamagawa \\ Department of Human and Information Systems, Faculty of Engineering, Gifu University, Gifu, Japan
}

\begin{abstract}
Unlike well-investigated polymer-based soft actuators such as gel, Ionic Polymer-Metal Composite, conducting polymer, a CFRP-based polymeric laminate possesses quite distinguished properties. It was previously reported that a CFRP-PVC laminate exhibited two-way deflection in accordance with environmental temperature, but it could not exhibit symmetric deflection because of its asymmetric structure. It must broaden the potential usefulness of the CFRP-based polymeric laminate as a soft actuator by achieving its symmetric deflection. In this study, we attempted to fabricate a new CFRP-based polymeric laminate which could exhibit symmetric deflection by electrical laminate temperature control. Despite such a successful outcome, still there was a room to improve the performance of the new CFRP-based polymeric laminate especially about the degree of its deflection. Its deflection was not so large for the actuator use. Hence, we further attempted to fabricate another CFRP-based polymeric laminate which could exhibit large and symmetric deflection under electrical control of laminate temperature. In fact, this another CFRP-based laminate exhibited symmetric deflection in accordance with temperature and the deflection was indeed enhanced.
\end{abstract}

Keywords: CFRP, PVC, actuator, laminate, deflection

\section{Introduction}

Soft actuators have attracted considerable attention of researchers and engineers. Recently, research on electroactive polymer-based soft actuators has become widespread, and hydrogels [1,2], ionic polymermetal composites (IPMC) [3-5], and conducting polymers [6,7] are being intensively investigated. These polymers have unique characteristics that are useful for actuator use. However, some of their properties are undesirable. For example, hydrogel shape change speed is considerably slow, IPMC hardly generates high force, and conducting polymers are typically electrically activated in a highly wet state. A practical soft actuator should exhibit rapid shape change and generate high force, Furthermore, the use of a liquid is not preferred because of the difficulty in handling a wet actuator.

A CFRP-PVC laminate is a polymeric laminate that is fabricated simply by gluing a carbon fiber reinforced plastic (CFRP) plate to a polyvinylchloride (PVC) plate [8]. CFRP causes Joule heating by the application of voltage. Because of the disparity in the coefficients of thermal expansion between CFRP and PVC, the CFRP-PVC laminate exhibits deflection by imposing a voltage on the CFRP portion. Therefore, the CFRP-PVC laminate is regarded as an electroactive polymer, though its deflection is thermally induced. Shape change of the CFRP-PVC laminate in response to the external electric stimulation considerably rapid, and the CFRP-PVC laminate can generate an adequately high force. Moreover,

\footnotetext{
${ }^{*}$ Corresponding author: Minoru Sasaki, Department of Human and Information Systems, Faculty of Engineering, Gifu University, 1-1 Yanagido, Gifu, 501-1193 Japan. Tel.: +81 58293 2541; Fax: +81 58293 2541; E-mail: sasaki@ gifu-u.ac.jp.
}

1383-5416/13/\$27.50 (C) 2013 - IOS Press and the authors. All rights reserved

This article is published online with Open Access and distributed under the terms of the Creative Commons Attribution NonCommercial License. 


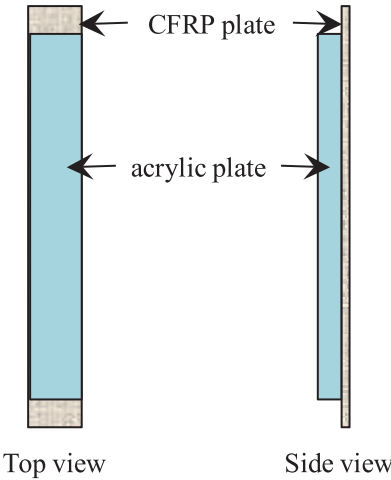

Fig. 1. Structure of AClaminate.

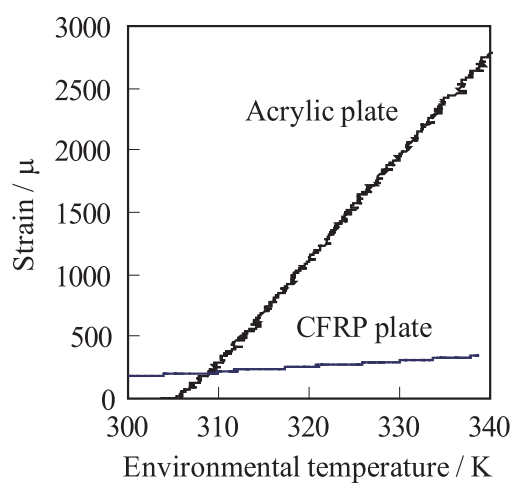

Fig. 2. Strain vs. environmental temperature.

it is a solid-state material. Although the CFRP-PVC laminate possesses useful characteristics as a soft actuator, it has some limitations. For instance, the CFRP-PVC laminate assumes a deflected shape in the PVC plate direction at room temperature and the Joule heat generated by the voltage application straightens the CFRP-PVC laminate. However, because of the asymmetric structure of CFRP-PVC laminate, the CFRP-PVC laminate hardly deflects in the CFRP plate direction. That is, the CFRP-PVC laminate exhibits asymmetric rather than symmetric deflection. Therefore, in this study, we attempted to fabricate a polymeric laminate-type actuator that could exhibit large and symmetric deflection, and quantitatively evaluated its actuator performance.

\section{Background}

Coefficient of thermal expansion of CFRP is quite small compared with that of well-known polymers such as PVC, acrylic plate and so on. Therefore, a laminate fabricated by gluing such a well-known polymer plate to the CFRP plate results in a thermally deformable laminate, that is a bimorph actuator. We fabricated an Acrylic plate-CFRP plate laminate (hereafter called AClaminate for short), where its structure is illustrated in Fig. 1. Since the coefficient of thermal expansion of acrylic plate is quite high compared with that of CFRP plate as clearly indicated by the experimental results of Strain of Acryl plate and CFRP plate vs. Environmental temperature shown in Fig. 2, the AClaminate was expected to deflect by being heated.

Figure 3 shows an illustration of the experimental setup for measuring the tip displacement of the AClaminate. The AClaminate was vertically clamped. Both ends the CFRP plate of the AClaminate were connected to a power supply. Immediately after a constant voltage was imposed on the CFRP plate, Joule heat was generated. The Joule heat caused a larger expansion of the acrylic plate than the CFRP plate, resulting in the deflection of the AClaminate in the direction indicated by the dashed arrow in Fig. 3. Turning off the voltage restored the AClaminate to its initial straight shape because of natural convection cooling. Tip displacement of the AClaminate was measured with a laser displacement sensor as a function of time. At the same time, the surface temperature of the AClaminate was measured with radiation thermometers as a function of time, too. Photos of the AClaminate during deflection and shape restoration is shown in Fig. 4. Because of the asymmetric structure of AClaminate, the AClaminate could not exhibit symmetric shape change by the applied voltage control. The AClaminate always deflects in the direction indicated by the dashed arrow shown in Fig. 3, when it is under a voltage, but never 


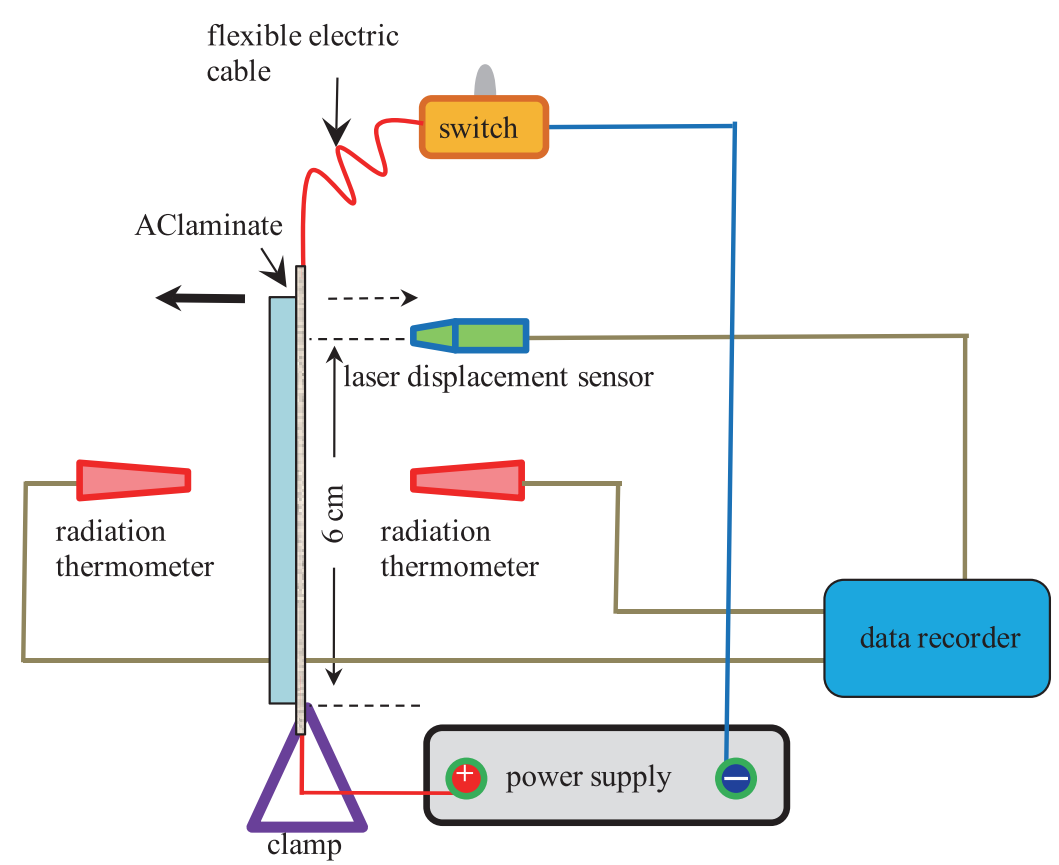

Fig. 3. Experimental setup for measuring the tip displacement of the AClaminate.

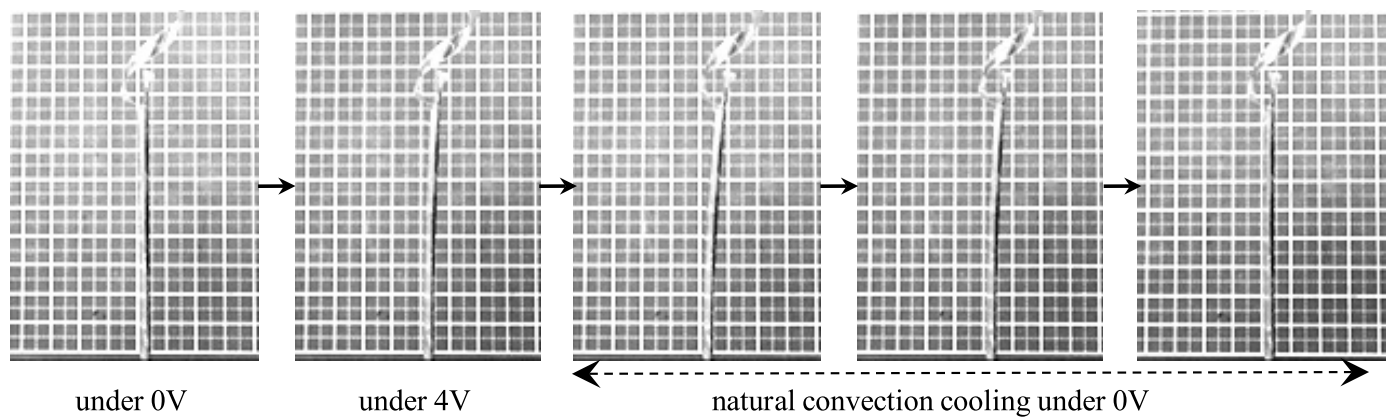

Fig. 4. Sequential photos of the shape change of AClaminate under applied voltage.

deflects in the direction indicated by a fat solid arrow shown in Fig. 3. Induction of the symmetric shape change of AClaminate must broaden its usefulness as a bending mode soft actuator. In order to achieve the symmetric shape change, the structure of AClaminate should be modified into symmetric structure as illustrated in Fig. 5. With this concept in mind, we fabricated symmetrically deflectable laminate consisting primarily of acrylic plates and a CFRP plate and quantitatively assessed its performance.

\section{Fabrication of symmetrically deflectable laminate}

The laminate whose structure is same as Fig. 5 cannot be deflected by imposing a voltage on the CFRP plate of the laminate. Since the Joule heat generated will heat up the both acrylic plates of the laminate at the same level, no deflection is expected. Therefore the temperature of individual acrylic plates should be maintained different each other. We fabricated a CFRP-based polymeric laminate by 


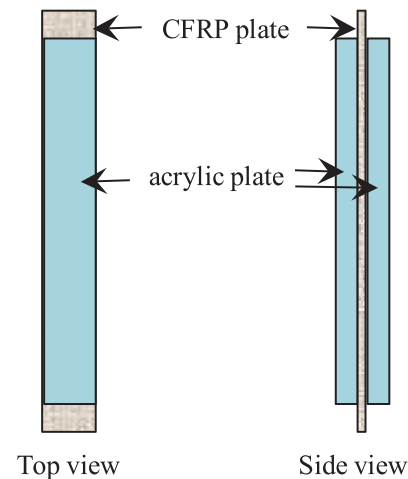

Fig. 5. Structure of symmetrically deflectable CFRP-based polymeric laminate.

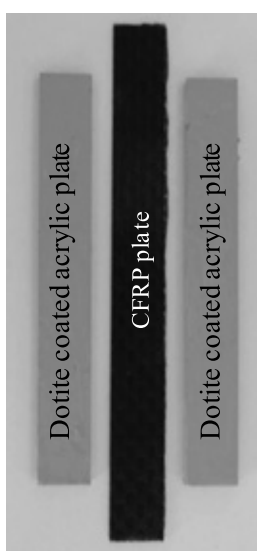

(a)

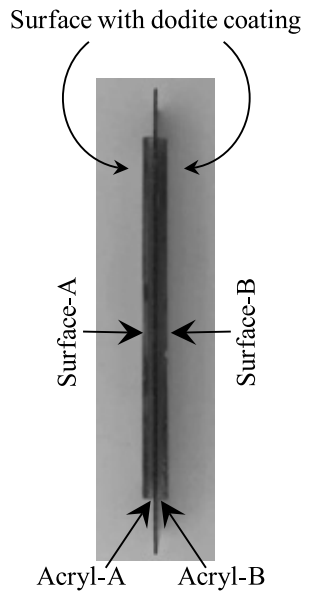

(b)

Fig. 6. (a) Ingredients of ACAlaminate (b) ACAlaminate.

the following procedure: Two acrylic plates were glued on a CFRP plate, and one surface of individual acrylic plates were coated with an electrically conductive paint, dotite (Fujikura Kasei Co., Ltd., Japan) as shown in Fig. 6(a). The dotite coating served as a flexible electrode, and it also served as a resitor for generating Joule heat, too. These plates were assembled into one as shown in Fig. 6(b) using an instant glue. The resultant laminate was designated as ACAlaminate. For the convenience of following discussion, individual dotite coatings were designated as Surface-A and Surface-B, respectively, and the acrylic plates on which Surface-A and Surface-B were respectively designated as Acryl-A and Acryl-B as shown in Fig. 6(b).

\section{Measurement}

Tip displacement of the ACAlaminate under thermal control by controlling the voltage imposed on the dotite coatings (Surface-A, Surface-B) was measured using a laser displacement sensor as a function of time. At the same time, temperatures of Surface-A and Surface-B were measured with radiation thermometers.

\section{Results and discussion}

\subsection{Deflection of ACAlaminate}

The tip deflection of ACAlaminate under $4.5 \mathrm{~V}$ was measured using the setup shown in Fig. 3, where the electrical cables were connected to the Surface-A and Surface-B so as to be able to impose a voltage on the dotite coatings. The surface temperatures of both Surface-A and Surface-B were measured simultaneously, too. Once the voltage $4.5 \mathrm{~V}$ was imposed on Surface-A, the ACAlaminate deflects toward Surface-B direction due to the thermal expansion of the Acryl-A and vice versa once the voltage was imposed on Surface-B. Figure 7 shows time dependence of curvature of the ACAlaminate. Obviously symmetric deflection was induced by imposing voltage on Surface-A or Surface-B. However 


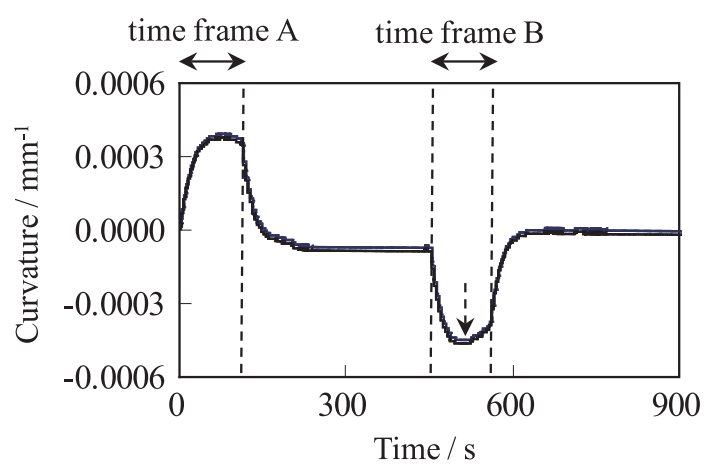

Fig. 7. Time dependence of ACAlaminate curvature under the voltage of $4.5 \mathrm{~V} 4.5 \mathrm{~V}$ was imposed on Surface-A during time frame A, while it was imposed on Surface-B during time frame B.

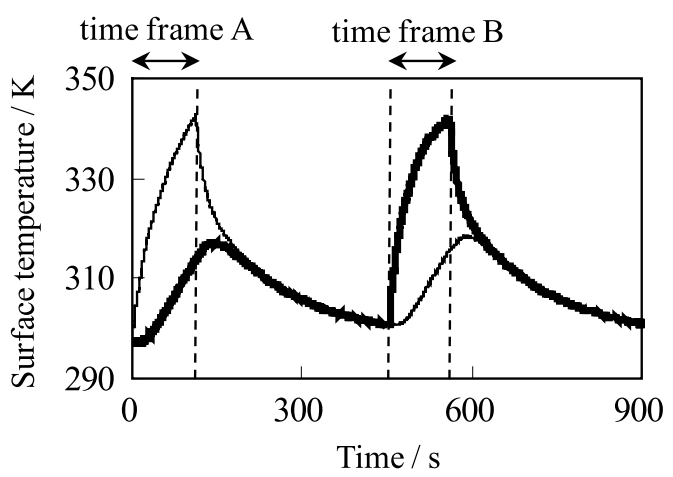

(a)

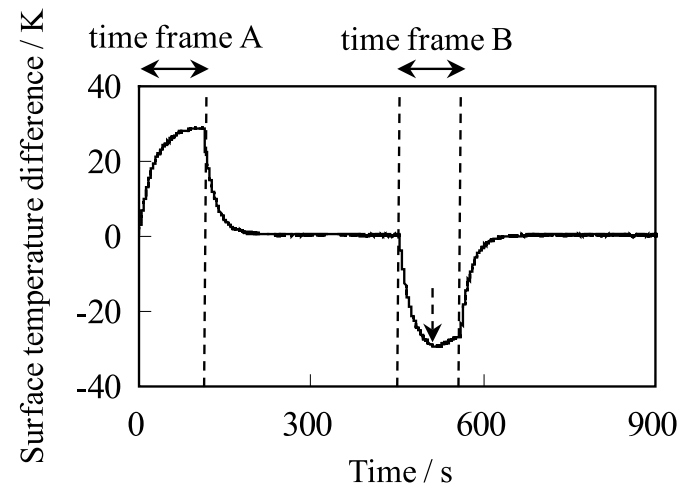

(b)

Fig. 8. Time dependence of ACAlaminate temperature, when obtaining the data of Fig. 7 (a) Fine line: the temperature of Surface-A Fat line: the temperature of Surface-B (b) Temperature of Surface-A in reference to that of the Surface-B.

the deflection curvature was not so large. Even though the voltage was imposed, for example, only on the Surface-A for long while, larger deflection was not observed, since the Joule heat generated at the Surface-A gradually conducted to Acryl-B through the Acryl-A and the CFRP plate. Therefore, the temperature difference between the Acryl-A and Acryl-B could not be maintained large enough for so long while, resulting in the decay of deflection curvature. Figure 8(a) shows surface temperature of the ACAlaminate, and Fig. 8(b) shows the temperature difference between the Surface-A and Surface-B, where the temperature of Surface-B was reference temperature. For example, the moment indicated by dashed arrow in Fig. 8(b), the temperature difference decayed, although the voltage was imposed on the Surface-B at this moment. It is due to the heat conduction to Acryl-A through Acryl-B and the CFRP plate. In fact, the surface temperature of acrylic plate of the Surface-A gradually increased as seen in Fig. 8(a), though the voltage was not imposed on the Surface-A at all at this moment. Therefore the temperature difference between the Surface-A and the Surface-B started gradually decaying even under $4.5 \mathrm{~V}$ as indicated by dashed arrow in Fig. 8(b). Therefore, the curvature started decaying at the moment indicated by dashed arrow in. Figure 7 Therefore it is necessary to achieve large temperature difference in order to achieve and maintain large deflection. 

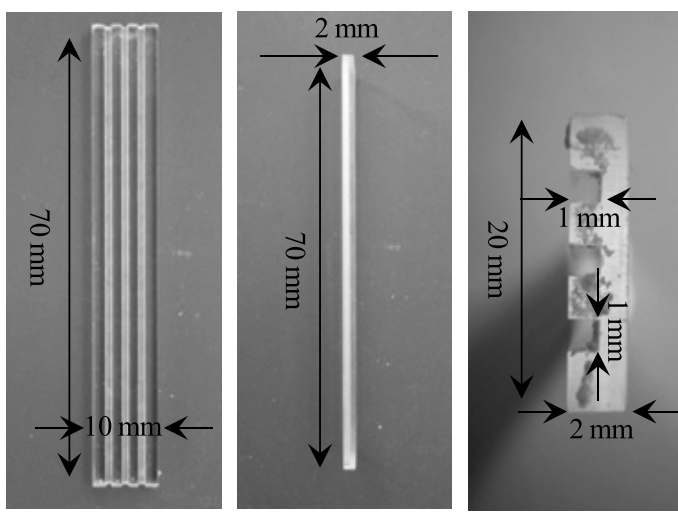

Fig. 9. An acrylic plate with three grooves.

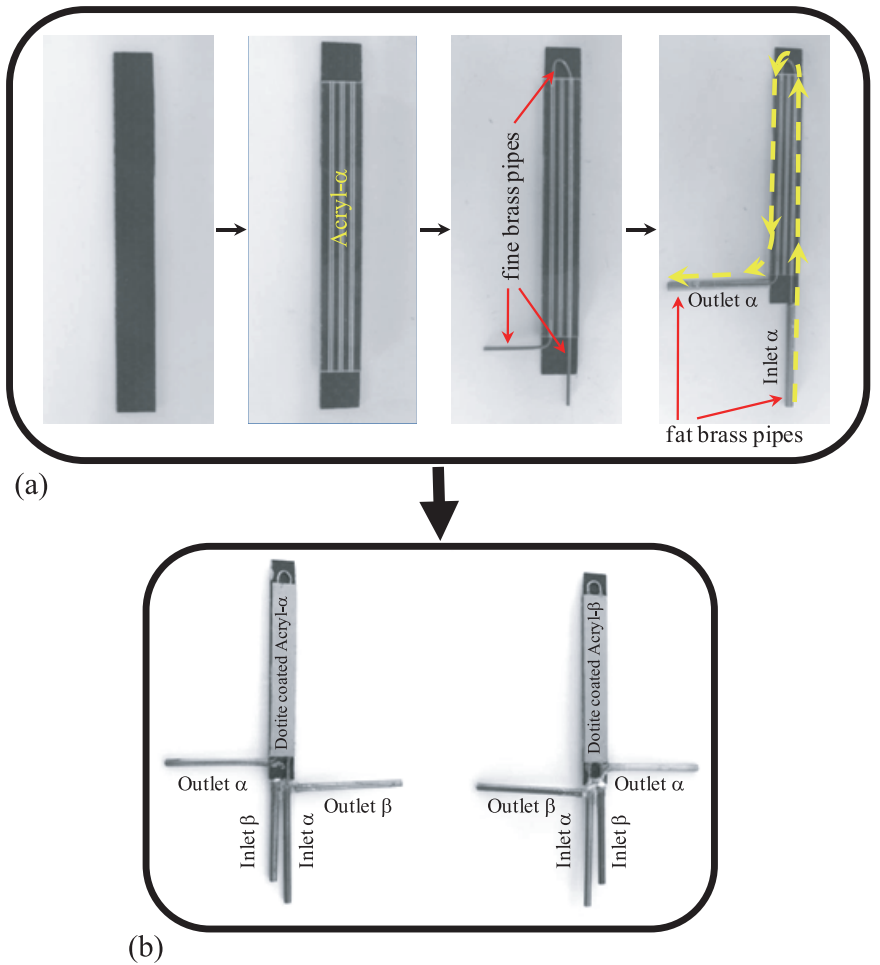

Fig. 10. (a) Fabrication process of a new CFRP-based laminate (ACAlaminate2) (b) Entire structure of ACAlaminate2.

\subsection{Improvement of ACAlaminate deflection}

A new CFRP-based polymeric laminate was fabricated in order to realize large and symmetric deflection. Two pieces of grooved acryl plates whose structure is shown in Fig. 9 were prepared first. A piece of them was glued on a CFRP plate as shown in Fig. 10(a), where the grooved surface faced the CFRP plate. This acrylic plate was designated as Acryl- $\alpha$. Three channels were formed between the Acryl- $\alpha$ and the CFRP plate. Three fine brass pipes were inserted into the channels; one of which was U-shaped and connected the right and left channels as shown in Fig. 10(a). Two thick brass pipes were placed over the fine brass pipes at the bottom of the laminate. The brass pipes connected the right channel to the left channel, forming a single channel as indicated by the dashed arrows in Fig. 10(a). The two brass pipes at the bottom were respectively designated as Inlet- $\alpha$ and Outlet- $\alpha$ as shown in Fig. 10(a). The same structure was created at the opposite side of the CFRP plate, and the acryl plate glued to this side was designated as Acryl- $\beta$. The two fat brass pipes at the bottom were respectively designated as Inlet $-\beta$ and Outlet- $\beta$ Fig. 10(b). The resultant laminate is hereafter called ACAlaminate2. Both Acryl- $\alpha$ and Acryl- $\beta$ were coated with dotite, although voltage would not be imposed on the ACAlaminate 2 for the deflection curvature measurement described next. The dotite coatings were applied on the ACAlaminate 2 merely for fabricating it under the same fabrication condition of ACAlaminate as far as possible.

Curvature of ACAlaminate 2 was measured under the individual temperature control of Acryl- $\alpha$ and Acryl- $\beta$ by supplying either hot water $\left(75^{\circ} \mathrm{C}\right)$ or cold water $\left(15^{\circ} \mathrm{C}\right)$ from Inlet- $\alpha$ and Inlet $\beta$. Although ACAlaminate 2 required the use of a liquid (water), the water was completely confined in ACAlaminate2; therefore, ACAlaminate 2 was not in the wet state. Hence, the use of water was not an obstacle in the 


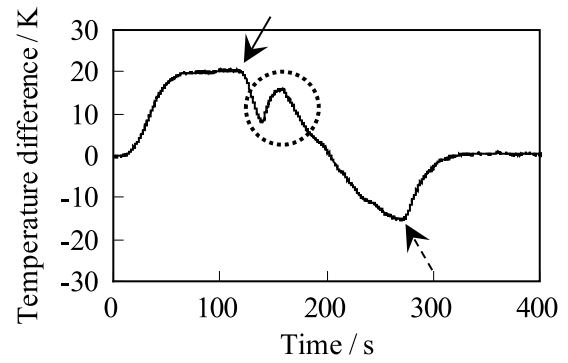

(a)

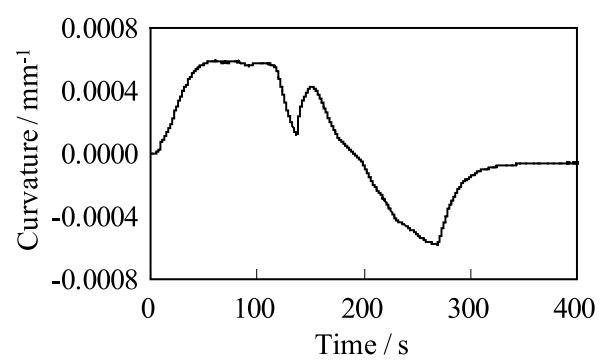

(b)

Fig. 11. (a) Time dependence of the Acryl- $\alpha$ temperature in reference to that of Acryl- $\beta$ (b) Time dependence of ACAlaminate2 curvature.

use of ACAlaminate2, unlike the previously mentioned conducting polymer-based actuator. Figure 11(a) shows the time dependence of the Acryl- $\alpha$ temperature in reference to that of Acryl- $\beta$. Initially, hot water was circulated from Inlet- $\alpha$ through Outlet- $\alpha$, while cold water was circulated from Inlet- $\beta$ through Outlet- $\beta$. Hence, the surface temperature difference increased. The curvature of ACAlaminate 2 is shown in Fig. 11(b), which increased and reached a high of $0.00055 \mathrm{~mm}^{-1}$, higher than the peak curvature of ACAlaminate shown in Fig. 7. Thus, an effective temperature difference was realized in ACAlaminate2. Cold water was circulated from Inlet- $\alpha$ through Outlet- $\alpha$ at the time indicated by the solid arrow in Fig. 11(a), while hot water was circulated from Inlet- $\beta$ through Outlet- $\beta$. Then, the curvature of ACAlaminate 2 started decreasing. We intentionally induced a temperature disturbance in ACAlaminate2 by controlling the flow rate of hot water as indicated by the dotted circle in Fig. 11(a). The curvature of ACAlaminate 2 was disturbed by the temperature change, and it passed through zero curvature and continued decreasing. Its lowest peak was at $-0.00053 \mathrm{~mm}^{-1}$, and its magnitude was almost same as that of the highest peak. Therefore, it is concluded that symmetric deflection was successfully achieved. Natural convection cooling was allowed from the time indicated by the dashed arrow in Fig. 11(a). The deflection of ACAlaminate2 perfectly followed the temperature behavior. Such a strong obedience of the ACAlaminate 2 curvature to the temperature difference was observed even when the temperature disturbance was applied.

\section{Conclusion}

A CFRP-based polymeric laminate-type actuator that exhibited symmetric deflection was fabricated by gluing two acryl plates to both sides of a CFRP plate. It was experimentally confirmed that symmetric deflection of the actuator could be achieved by controlling the temperature of individual layers of the laminate. Circulation of both hot and cold water through the CFRP-based laminate-type actuator enabled the appropriate control of the laminate temperature, resulting in its symmetric deflection. Although a liquid (water) was employed to control the laminate temperature, the liquid was completely confined in the laminate. Therefore, water was not an obstacle in the use of the CFRP-based laminate, unlike the wet-type actuator.

\section{Acknowledgments}

This work was financially supported by Ministry of Education, Culture, Sports, Science and Technology for the financial support under the Knowledge Cluster Initiative the Second Stage Tokai Region 
Nanotechnology Manufacturing Cluster and the Air Force Research Laboratory under the agreement number FA23861014119.

\section{References}

[1] T. Tanaka, I. Nishio, S.T. Sun and S. Ueno-Nishio, Collapse of gels under an electric field, Science 218 (1982), 467-469.

[2] Y. Osada, H. Okuzaki and H. Hori. A polymer gel with electrically driven motility, Nature 355 (1992) 242-244.

[3] K. Oguro, Y. Kawami and H. Takenaka, Bending of an ion-conducting polymer film-electrode composite by an electric stimulus at low voltage, Trans J Micromach Soc 5 (1992), 27-30.

[4] K. Oguro, K. Asaka and H. Takenaka, Polymer film actuator driven by low voltage, in Proceedings of 4th International Symposium on Micro Machine and Human Science, Nagoya, (1993), 39-40.

[5] M. Sasaki, Y. Onouchi, T. Ozeki, H. Tamagawa and S. Ito, Feed forward control of an ionic polymer-metal composite actuator, International Journal of Applied Electromagnetics and Mechanics 33 (2010), 875-881.

[6] K. Sugiyama, K. Ishii and K. Kaneto, A control method of a biomimetic actuator using electroconductive polymer, in Proceeding of SCIS \& ISIS, Nagoya, (2008), 1687-1692.

[7] A.S. Lee, S.F. Peteu1, J.V. Ly, A.A.G. Requicha, M.E. Thompson and C. Zhou, Actuation of polypyrrole nanowires, Nanotechnol 19 (2008), 165501.

[8] H. Tamagawa, K. Kikuchi and G. Nagai, Mechanical characteristics of a thermo-responsive two-way shape change polymeric laminate, Sens Actuators A: Physical 163 (2010), 356-362. 